\title{
Satisfação com atendimento e intenção de amamentação de gestantes em uma instituição pública
}

\author{
Satisfaction with treatment and intention to breastfeed of pregnant \\ women in a public institution \\ Satisfacción con atención e intención de lactancia de gestantes \\ en una institución pública
}

\author{
Tânia Adas SALIBA \\ Aryane Kame TAMANAHA \\ Denise Toledo Rós \\ Cléa Adas Saliba GARBIN \\ Suzely Adas Saliba MOIMAZ
}

\begin{abstract}
Programa de Pós-Graduação em Odontologia Preventiva e Social, Faculdade de Odontologia de Araçatuba Univ Estadual Paulista, UNESP 16015.050 Araçatuba-SP, Brasil
\end{abstract}

\begin{abstract}
Resumo
Introdução: O atendimento odontológico na gestação foi incluído no pré-natal regular das gestantes, no Sistema Único de Saúde no Brasil, a partir do ano de 2010, sendo a avaliação do serviço prestado de grande importância para melhorar a qualidade do serviço e observar até que ponto os objetivos estabelecidos foram alcançados, de acordo com a perspectiva das bases populacionais às quais se dirige. Objetivo: Verificar a qualidade dos serviços prestados no Programa de Atenção Odontológica à Gestante e a intenção de amamentação das gestantes. Método: Trata-se de uma pesquisa transversal, retrospectiva, com gestantes que concluíram o tratamento odontológico em instituição pública, no período de 2014 a 2016, em Araçatuba-São Paulo. Foram incluídas 124 gestantes, as quais responderam um questionário de avaliação sobre o serviço prestado e sua intenção de amamentação. Os dados obtidos foram digitados e analisados com auxílio do programa Epi Info 7.2.2 (Center for Disease Control and Prevention). Resultados: Das gestantes participantes da pesquisa, classificaram como ótimo $91,13 \%$ a apresentação pessoal e atendimento clínico e $91,94 \%$ a gentileza, 90,32\% sentiram segurança nas orientações recebidas, e 98,38\% sentiram-se seguras durante o tratamento. Com relação a amamentação, 76,61\% afirmaram que pretendiam amamentar seus bebês. Conclusão: A qualidade do Programa de Atenção Odontológica pode ser considerada satisfatória e grande parte das gestantes possuia intenção de amamentar seu bebê.

Descritores: Gestantes; Assistência Odontológica; Pesquisa sobre Serviços de Saúde.
\end{abstract}

\begin{abstract}
Introduction: Dental care during gestation was included in the regular prenatal care of pregnant women, in the Brazilian Unified Health System, starting in 2010, and the evaluation of the service provided was of great importance to improve the quality of the service and to observe until that the established objectives were reached, according to the perspective of the population bases to which it is directed. Objective: To verify the quality of the services provided in the Program of Dental Care to the Pregnant Woman and the intention to breastfeed the pregnant women. Method: This is a cross-sectional, retrospective study with pregnant women who completed dental treatment in a public institution, from 2014 to 2016, in Araçatuba-São Paulo. A total of 124 pregnant women were included, who answered an evaluation questionnaire about the service provided and their intention to breastfeed. The data were typed and analyzed using the Epi Info 7.2.2 program (Center for Disease Control and Prevention). Results: Of the pregnant women who participated in the study, $91.13 \%$ presented a personal presentation and clinical care, $91.94 \%$ were kind, $90.32 \%$ felt safety in the guidelines received, and $98.38 \%$ felt safe during treatment. Regarding breastfeeding, $76.61 \%$ stated that they intended to breastfeed their babies. Conclusion: The quality of the Dental Care Program can be considered satisfactory and most of the pregnant women intend to breastfeed their baby.

Descriptors: Pregnant Women; Dental Care; Health Services Research.
\end{abstract}

\section{Resumen}

Introducción: La atención odontológica en la gestación fue incluida en el prenatal regular de las gestantes, en el Sistema Único de Salud en Brasil, a partir del año 2010, siendo la evaluación del servicio prestado de gran importancia para mejorar la calidad del servicio y observar hasta que los objetivos establecidos se alcanzaron, de acuerdo con la perspectiva de las bases poblacionales a las que se dirige. Objetivo: Verificar la calidad de los servicios prestados en el Programa de Atención Odontológica a la Gestante y la intención de amamantar de las gestantes. Método: Se trata de una investigación transversal, retrospectiva, con gestantes que concluyeron el tratamiento odontológico en institución pública, en el período de 2014 a 2016, en Araçatuba-São Paulo. Se incluyeron 124 gestantes, las cuales respondieron un cuestionario de evaluación sobre el servicio prestado y su intención de lactancia. Los datos obtenidos fueron digitados y analizados con ayuda del programa Epi Info 7.2.2 (Center for Disease Control and Prevention). Resultados: De las gestantes participantes de la investigación, clasificaron como óptimo 91,13\% la presentación personal y atención clínica y 91,94\% la gentileza, el 90,32\% sintió seguridad en las orientaciones recibidas, y el 98,38\% se sintió seguro durante el tratamiento. Con respecto a la lactancia, el 76,61\% afirmó que pretendían amamantar a sus bebés.Conclusión: La calidad del Programa de Atención Odontológica puede ser considerada satisfactoria y gran parte de las gestantes tuvieron intención de amamantar a su bebé.

Descriptores: Mujeres Embarazadas; Atención Odontologica; Investigación em Servicios de Salud.

\section{INTRODUÇÃO}

O atendimento odontológico na gestação foi incluído no pré-natal regular das gestantes, no Sistema Único de Saúde (SUS) no Brasil, a partir do ano de 2010, no entanto estudos mostraram que a taxa de consultas odontológicas na gestação ainda é baixa ${ }^{1-3}$.

De modo geral, existem poucas informações sobre o uso de serviços odontológicos por parte da população brasileira, e de acordo com dados da Pesquisa Nacional de Saúde, realizada pela Fundação Instituto Brasileiro de Geografia e Estatística (IBGE) em 2010, cerca de 44,4\% da população brasileira consultou um dentista há menos de um ano da data de entrevista, As Regiões Norte e Nordeste apresentaram as menores proporções deste indicador $(34,4 \%$ e $37,5 \%$, respectivamente). A proporção de pessoas que consultou o cirurgião-dentista foi superior à média nacional para mulheres $(47,3 \%)$ evidenciando a existência de um entrave para o acesso ao atendimento odontológico ${ }^{4,5}$.

Com relação à baixa taxa de atendimento odontológico durante a gestação, algumas barreiras são apontadas: a dificuldade de acesso ao tratamento, o medo, ansiedade, insegurança, crenças e tabus ${ }^{5,6}$.

A gravidez é um período da vida da mulher em que ocorrem alterações fisiológicas e psicológicas, podendo interferir na saúde bucal e no desenvolvimento de problemas sistêmicos. Essas alterações predispõem o agravamento de processos inflamatórios periodontais ou surgimento de patologias inexistentes na cavidade bucal antes da gestação ${ }^{7}$. Por outro lado, também proporcionam uma etapa favorável 
para a promoção de saúde, devido a série de dúvidas que podem estimular a gestante a buscar informações, possibilitando a incorporação de mudanças de hábitos, e, com isso, adquirir novas e melhores práticas de saúde ${ }^{8}$.

Durante a gravidez, a manutenção da saúde bucal tem sido reconhecida como um desafio para a Saúde Pública, pois há registros de alta frequência de inflamação gengival entre gestantes ${ }^{9}$. O Ministério da Saúde recomenda, em seu manual de assistência à saúde durante o pré-natal, cuidados odontológicos para garantir o bem-estar da gestante e orientála quanto aos cuidados necessários ${ }^{10}$.

Considerando a importância da manutenção da saúde bucal e sua relação com a saúde geral, em 1998 foi implantado na FOA-UNESP, o Programa de Atenção Odontológica à Gestante, integrado ao serviço municipal de saúde, objetivando o cuidado integral, com o desenvolvimento de ações educativo-preventivas e de recuperação da saúde das gestantes incluídas no pré-natal do SUS.

A avaliação do serviço prestado, nas suas diferentes dimensões, faz-se necessária, pois permite melhorar a qualidade, na perspectiva das bases populacionais às quais se dirige $^{11}$. Tem como característica a atribuição de valor, verificando o cumprimento de objetivos, potencializando a eficiência, eficácia e efetividade das estruturas, processos e resultados, relacionados ao risco, acesso e satisfação dos usuários em busca de resolubilidade e qualidade ${ }^{12,13}$.

Um dos objetivos da política nacional de atenção integral à saúde da mulher é ampliar, qualificar e humanizar a atenção integral à saúde da mulher no $\mathrm{SUS}^{14}$. A Atenção Básica deve buscar a atenção integral e de qualidade, resolutiva e que contribua para o fortalecimento da autonomia das pessoas no cuidado à saúde, estabelecendo articulação orgânica com o conjunto da rede de atenção à saúde ${ }^{15}$. Todo serviço de saúde deve estabelecer, como rotina, a busca ativa das gestantes de sua área de abrangência. Para isso, os profissionais de saúde bucal devem trabalhar de forma integrada com os demais profissionais da atenção básica, tanto nas atividades educativas em grupo, como no acompanhamento de pré-natal. Espera-se a ocorrência de, no mínimo, uma avaliação odontológica a cada trimestre de gestação $^{16}$. Cabe ao SUS ter uma rede integrada que promova atenção qualificada e contínua aos indivíduos e às coletividades, de forma equitativa, garantindo universalidade, integralidade, equidade, participação social e descentralização ${ }^{17}$.

Dessa forma, avaliar os programas de atenção à saúde, possibilita observar até que ponto os objetivos estabelecidos foram alcançados, bem como permite analisar as expectativas dos usuários e dos profissionais de saúde, as barreiras enfrentadas no atendimento odontológico, visando a melhoria do serviço ofertado. O objetivo desta pesquisa foi verificar a qualidade dos serviços prestados no Programa de Atenção Odontológica à Gestante e a intenção de amamentação das gestantes.

\section{MATERIAL E MÉTODO}

Trata-se de uma pesquisa transversal, retrospectiva, com gestantes que concluíram o tratamento odontológico em instituição pública, pertencente ao SUS.

Foram incluídas 124 gestantes, as quais responderam completamente questionários de avaliação sobre o serviço de saúde nos anos de 2014 a 2016 no município de Araçatuba, São Paulo. O instrumento continha questões fechadas e abertas sobre a infraestrutura, satisfação, intenção de amamentação e sentimentos como medo e insegurança do atendimento no programa. As questões fechadas eram relacionadas à infraestrutura, atendimento e características dos profissionais, e as respostas permitiam atribuição de conceitos de "fraco" a "ótimo". As questões sobre medo, segurança, aprendizado, satisfação e amamentação tinham como opção de resposta: "sim" ou "não". As questões abertas abordavam conhecimento sobre o programa, ocupação e idade da gestante.

As variáveis dependentes analisadas nessa pesquisa foram: intenção de amamentação, satisfação com o atendimento, além de outras, como: idade, escolaridade, ocupação, indicação para o acesso ao programa, motivos para consulta, infraestrutura, características do profissional, medo, insegurança, aprendizado.

Apenas as gestantes com alta no tratamento odontológico foram convidadas voluntariamente para participarem da pesquisa. Pacientes que não concluíram o tratamento odontológico foram excluídas do estudo.

Os dados obtidos foram digitados no programa Epi Info 7.2.2 (Center for Disease Control and Prevention) e então realizadas as análises estatísticas descritivas.

\section{RESULTADOS}

Dentre as gestantes que participaram da pesquisa, $66,13 \%$ estavam na faixa etária de 21 à 35 anos de idade; $54,03 \%$ haviam completado o ensino médio e $63,71 \%$ estavam ativas no trabalho (Tabela 1 ).

Tabela 1. Distribuição numérica e percentual de gestantes segundo dados sociodemográfico $(\mathrm{n}=124)$. Araçatuba, 2017

\begin{tabular}{llll}
\hline Características & $\mathbf{n}$ & $\%$ \\
\hline Idade & $16-20$ & 19 & 15,32 \\
& $21-25$ & 25 & 20,16 \\
& $26-30$ & 24 & 19,36 \\
& $31-35$ & 33 & 26,61 \\
& $36-40$ & 14 & 11,29 \\
& $41-45$ & 5 & 4,03 \\
& Não respondeu & 4 & 3,23 \\
\hline Escolaridade & Ensino fundamental incompleto & 8 & 6,45 \\
& Ensino fundamental completo & 6 & 4,84 \\
& Ensino médio incompleto & 9 & 7,26 \\
& Ensino médio completo & 67 & 54,03 \\
& Ensino superior incompleto & 7 & 5,64 \\
& Ensino superior completo & 18 & 14,52 \\
& Não respondeu & 9 & 7,26 \\
\hline Ocupação & Do lar & 27 & 21,77 \\
& Estudante & 5 & 4,03 \\
& Ativa & 79 & 63,71 \\
& Desempregada & 4 & 3,23 \\
& Não respondeu & 9 & 7,26 \\
\hline
\end{tabular}

As formas de conhecimento das gestantes sobre o Programa de Atenção odontológica à Gestante estão demonstradas na Figura 1 e os motivos pela procura pelo atendimento estão representados na Figura 2.

A Tabela 2 apresenta a opinião das gestantes com relação a infraestrutura da clínica do Programa de Atenção Odontológica à Gestantes. Não houve classificação nas categorias médio e regular. A maioria das gestantes classificou como ótimo a "apresentação pessoal", "gentileza", "segurança nas orientações do cirurgião-dentista" e "atendimento clínico recebido". Não houve classificação nas categorias médio, regular e fraco para qualquer uma das variáveis (Tabela 3 )

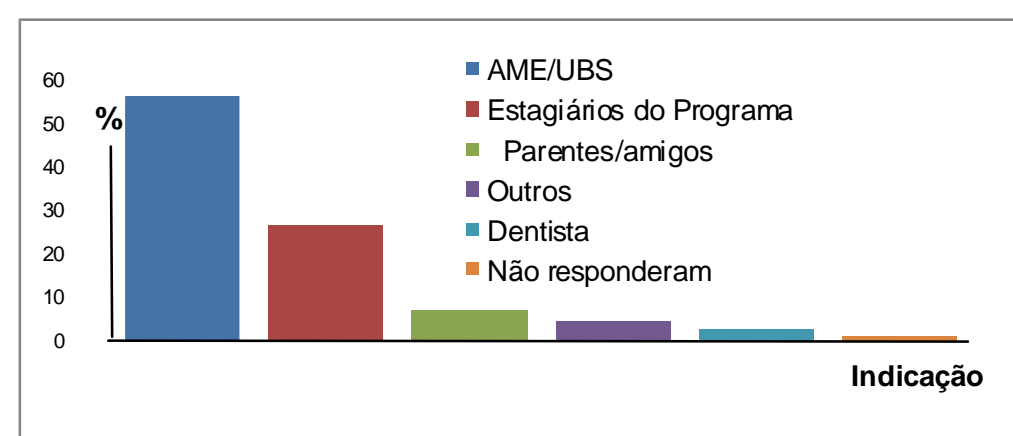

Figura 1: Distribuição numérica das gestantes de acordo com indicação para acesso ao Programa de Atenção Odontológica às Gestantes FOA UNESP. 


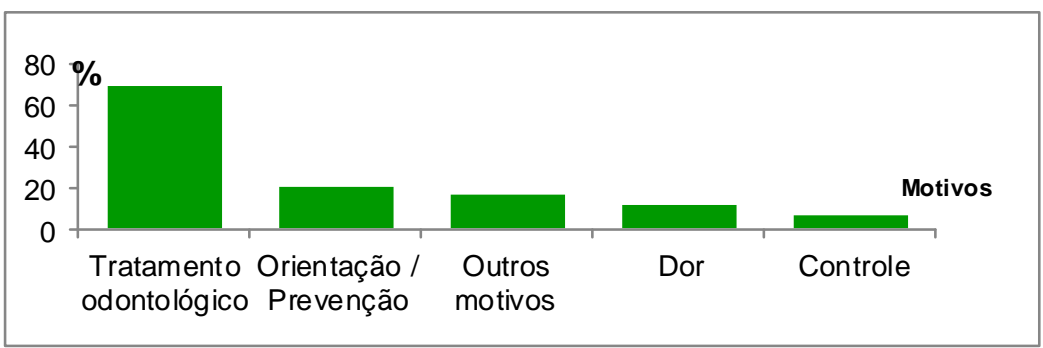

Figura 2: Distribuição percentual dos motivos pelos quais as gestantes procuraram atendimento no Programa de Atenção Odontológica às Gestantes da FOA/ UNESP.

Tabela 2. Distribuição numérica e percentual das respostas às variáveis relacionadas à infraestrutura da clínica, segundo o conceito atribuído. Araçatuba, 2017

\begin{tabular}{lrrrrrr}
\hline \multirow{2}{*}{$\begin{array}{l}\text { Conceito } \\
\text { Clínica }\end{array}$} & \multicolumn{2}{c}{ Limpeza } & \multicolumn{2}{c}{ Ventilação } & \multicolumn{2}{c}{ Conforto } \\
\cline { 2 - 7 } & \multicolumn{1}{c}{$\mathbf{n}$} & \multicolumn{1}{c}{$\%$} & \multicolumn{1}{c}{$\mathbf{n}$} & \multicolumn{1}{c}{$\%$} & \multicolumn{1}{c}{$\mathbf{n}$} & \multicolumn{1}{c}{$\%$} \\
\hline Ótimo & 109 & 87,90 & 103 & 83,06 & 100 & 80,64 \\
Bom & 14 & 11,29 & 18 & 14,52 & 21 & 16,94 \\
Fraco & 0 & 0,00 & 1 & 0,81 & 1 & 0,81 \\
Não opinou & 1 & 0,81 & 2 & 1,61 & 2 & 1,61 \\
Total & 124 & 100,00 & 124 & 100,00 & 124 & 100,00 \\
\hline
\end{tabular}

Tabela 3. Distribuição percentual e numérica das respostas às variáveis relacionadas ao profissional, segundo o conceito atribuído. Araçatuba,2017

\begin{tabular}{|c|c|c|c|c|c|c|}
\hline \multirow[b]{2}{*}{ Conceito Pessoal } & \multicolumn{2}{|c|}{ Ótimo } & \multicolumn{2}{|c|}{ Bom } & \multicolumn{2}{|c|}{ Total } \\
\hline & $n$ & $\%$ & $n$ & $\%$ & $n$ & $\%$ \\
\hline $\begin{array}{l}\text { Apresentação } \\
\text { pessoal }\end{array}$ & 113 & 91,13 & 11 & 8,87 & 124 & 100 \\
\hline Gentileza & 114 & 91,94 & 10 & 8,06 & 124 & 100 \\
\hline Atenção & 115 & 92,74 & 9 & 7,26 & 124 & 100 \\
\hline $\begin{array}{l}\text { Segurança } \\
\text { orientações }\end{array}$ & 112 & 90,32 & 12 & 9,68 & 124 & 100 \\
\hline $\begin{array}{l}\text { Atendimento clínico } \\
\text { recebido }\end{array}$ & 113 & 91,13 & 11 & 8,87 & 124 & 100 \\
\hline
\end{tabular}

Com relação ao medo durante o tratamento odontológico, 13,71\% das gestantes afirmaram que sentiram medo, entretanto $98,38 \%$ sentiram-se seguras. A maioria das gestantes mostrou-se satisfeita e indicaria o tratamento para outras (Tabela 4). Sobre amamentação, a maioria das gestantes afirmou que pretendia amamentar seus bebês e levalos ao dentista (Tabela 5).

Tabela 4. Distribuição numérica e percentual das gestantes, em relação às questões sobre o tratamento odontológico e a aprendizagem durante as atividades educativas $(\mathrm{n}=124)$. Araçatuba, 2017

\begin{tabular}{lcccccc}
\hline \multirow{2}{*}{ Questionamentos } & Sim & \multicolumn{3}{c}{ Não } & \multicolumn{3}{c}{ Não opinou } \\
\cline { 2 - 7 } & $\mathbf{n}$ & $\%$ & $\mathbf{n}$ & $\%$ & $\mathbf{n}$ & $\%$ \\
\hline $\begin{array}{l}\text { Sentiu medo durante o } \\
\text { tratamento } \\
\text { odontológico? }\end{array}$ & 17 & 13,71 & 106 & 85,48 & 1 & 0,81 \\
$\begin{array}{l}\text { Sentiu segurança no } \\
\text { tratamento? }\end{array}$ & 122 & 98,38 & 1 & 0,81 & 1 & 0,81 \\
$\begin{array}{l}\text { As dúvidas sobre o } \\
\text { tratamento odontológico } \\
\text { foram esclarecidas de } \\
\text { modo satisfatório? }\end{array}$ & 114 & 91,94 & 2 & 1,61 & 8 & 6,45 \\
$\begin{array}{l}\text { Houve aprendizagem } \\
\text { durante as reuniões } \\
\text { didático-pedagógicas? }\end{array}$ & 122 & 98,38 & 0 & 0,00 & 2 & 1,62 \\
$\begin{array}{l}\text { Você ficou satisfeita } \\
\text { com o atendimento? }\end{array}$ & 115 & 92,74 & 0 & 0,00 & 9 & 7,26 \\
$\begin{array}{l}\text { Você indicaria o } \\
\text { tratamento } \\
\text { odontológico? }\end{array}$ & 123 & 99,19 & 0 & 0,00 & 1 & 0,81 \\
\hline
\end{tabular}

Tabela 5. Distribuição numérica e percentual das gestantes, em relação às intenções das gestantes $(\mathrm{n}=124)$. Araçatuba, 2017

\begin{tabular}{|c|c|c|c|c|c|c|}
\hline \multirow[t]{2}{*}{ Questionamentos } & \multicolumn{2}{|l|}{ Sim } & \multicolumn{2}{|c|}{ Não } & \multicolumn{2}{|c|}{$\begin{array}{l}\text { Não } \\
\text { respondeu }\end{array}$} \\
\hline & $n$ & $\%$ & $n$ & $\%$ & $\mathbf{n}$ & $\%$ \\
\hline $\begin{array}{l}\text { Pretende amamentar seu/a } \\
\text { filho/a? }\end{array}$ & 95 & 76,61 & 1 & 0,81 & 28 & 22,58 \\
\hline $\begin{array}{l}\text { Pretende levar seu/a filho/a } \\
\text { a algum serviço destinado } \\
\text { ao atendimento } \\
\text { odontológico? }\end{array}$ & 89 & 71,78 & 11 & 8,87 & 24 & 19,35 \\
\hline
\end{tabular}

\section{DISCUSSÃO}

Nesta pesquisa sobre avaliação de serviço de saúde à gestante, foi verificado que, grande parcela delas pretendiam amamentar, tiveram suas dúvidas sanadas, e apesar de algumas apresentarem medo, sentiram-se seguras durante $o$ tratamento odontológico prestado. O cuidado à saúde das gestantes foi avaliado positivamente pelas mesmas.

Devido à grande importância da avaliação dos serviços, foi desenvolvido pelo governo brasileiro o PMAQ (Programa de Melhoria do Acesso e da Qualidade), com o objetivo de incentivar os gestores a melhorar a qualidade dos serviços de saúde oferecidos aos cidadãos nas Unidades Básicas de Saúde (UBS) ${ }^{18}$.

Durante 20 anos, o Ministério da Saúde brasileiro intensificou a produção de materiais de educação e informação em saúde voltados à temática do aleitamento materno, revelando a importância da informação e da comunicação no âmbito da saúde da criança ${ }^{19}$. Neste estudo, $30 \%$ das participantes não responderam ou não tinham intenção de amamentar, necessitando melhorias no cuidado na atenção básica, com práticas educativas e efetivas, com abordagem da importância do aleitamento materno, visando maior cuidado na atenção básica. A saúde bucal é parte inseparável da saúde geral e o aleitamento materno propicia o crescimento e desenvolvimento craniofacial adequado, pois a sucção para obtenção do leite materno proporciona um intenso exercício muscular, além de suprir as necessidades nutricionais do bebê, evitando a busca por chupeta e dedos ${ }^{20}$. Atividades de promoção da amamentação durante o período pré-natal podem levar a resultados positivos, por meio da conscientização e motivação da mulher ${ }^{21}$.

No âmbito da atenção odontológica, estudos apontam que quanto mais cedo a criança receber cuidados odontológicos, menor é a possibilidade do desenvolvimento da cárie dentária, por isso, a importância da atenção à saúde materno-infatil ${ }^{22,23}$. A consulta odontológica no pré-natal foi instituída no SUS e é um dos indicadores PMAQ, visando, principalmente, prevenir agravos de saúde bucal que possam comprometer a gestação e o bem-estar da gestante ${ }^{16}$. Na consulta odontológica, no pré-natal, bem como em ações voltadas às gestantes, integradas aos demais programas, devem ser incluídas atividades educativas sobre a importância do controle da placa bacteriana dentária, dos problemas bucais, e métodos de prevenção, como uso do flúor nas suas diferentes formas, selante oclusais, controle da dieta e outros. Além disso, é de fundamental importância, esclarecer sobre a segurança do tratamento odontológico no período gestacional, desmistificando-o, trabalhando as crenças e preocupações externadas pelas gestantes.

O Programa de Atenção Odontológica à Gestantes desde sua implantação é baseado no tripé ensino-pesquisaextensão, promovendo ações envolvendo alunos de graduação, pós-graduação e docentes da Faculdade de Odontologia de Araçatuba- FOA, UNESP, profissionais da saúde do município de Araçatuba e a comunidade, principalmente gestantes e familiares, público alvo do Programa.

Como porta de entrada para o Programa foram estabelecidas as Unidades de Saúde e o Ambulatório Médico de Especialidades (AME) do município. Em um primeiro momento, as gestantes participam de uma reunião didáticopedagógica, em forma de rodas de discussão, objetivando a desmistificação do atendimento odontológico na gestação e a conscientização da mulher sobre a saúde bucal. O programa de atenção à saúde da gestante inclui também duas reuniões, para discussão sobre aleitamento materno e saúde bucal do bebê. A partir do contato inicial dos graduandos e pósgraduandos, são realizadas atividades de educação em saúde na sala de espera, e as gestantes com necessidade de 
tratamento são encaminhadas para a clínica para posterior realização de procedimentos preventivos e curativos, visando a manutenção da saúde bucal.

Além de promover tratamento e conscientização das gestantes, o Programa aproxima os alunos de pós-graduação e graduação da universidade, docentes e funcionários da saúde, promovendo o desenvolvimento de pesquisas e produções cientificas e a maior capacitação profissional dos graduandos e pós-graduandos, para melhor atendimento desta parcela especial da população. Os discentes participantes do projeto, e os profissionais de saúde realizam treinamentos e capacitações para atuação no programa para aprofundamento sobre alterações fisiológicas na gestação, risco para afecções bucais e aleitamento materno. Segundo Moimaz et al., 2011, ações como desmistificar crenças e preocupações sobre a gravidez e o tratamento odontológico, conscientização a respeito dos problemas bucais, orientar-se sobre a importância do controle da placa, do uso de flúor, bem como cuidados com o futuro bebê, devem estar inclusas no pré-natal odontológico ${ }^{24}$.

Neste estudo, o medo esteve presente em poucas gestantes, e a grande maioria relatou segurança no atendimento recebido. Este achado pode ser devido ao caminho percorrido pela gestante no Programa, com a participação nas rodas de discussão, reuniões didáticopedagógicas, solucionando as dúvidas com relação ao atendimento odontológico durante a gestação, antes de ingressar na clínica.

A qualidade do serviço de saúde pode ser identificada, além de outros fatores, através de competência profissional, satisfação do usuário, acessibilidade, eficácia e eficiência ${ }^{25}$. Estudo anterior avaliando a humanização verificou que o programa atende aos princípios de humanização ${ }^{25}$. Os participantes do projeto devem exercer seu papel de educadores orientando as gestantes sobre as diversas mudanças que ocorrem neste período e reforçando a importância dos cuidados à saúde ${ }^{26}$.

A política humaniza SUS preconiza um atendimento mais digno e humanizado, considerando também, o ambiente e o conforto oferecidos, sendo possível melhora nas condições de trabalho e atendimento ${ }^{27}$.

Toma-se o cuidado para que as sessões clínicas não ultrapassem 40 minutos, a fim de não provocar desconforto nas pacientes, além do cuidado com a postura através da utilização de almofadas, evitando desta forma, a síndrome hipotensiva, esta síndrome é provocada pela compressão da veia cava, devido ao peso do útero em função do tamanho do feto nos últimos meses da gestação, a gestante então, deve ficar deslocada para o lado esquerdo, lado do coração, evitando a compressão dos vasos sanguíneos, evitando o desconforto. Alguns cirurgiões-dentistas muitas vezes se sentem despreparados para atender às gestantes, pois considera que elas são sempre pacientes de risco. Assim torna-se importante a necessidade de se modificar esse discurso, inserindo maiores informações sobre o assunto nos currículos de graduação e nos meios de comunicação do profissional, a fim de se alcançar uma ampla cobertura, desmistificando-se a crença e promovendo-se o aprendizado $^{28}$.

O medo dos profissionais de saúde bucal, de realizar tratamento odontológico na mulher, na fase gestacional, é também uma barreira para o cuidado integral da saúde da gestante. Mesmo sabendo que, tomando os cuidados necessários durante o atendimento odontológico, a maioria dos procedimentos pode ser realizada no período gestacional, há o temor de colocar em risco a saúde do bebê. Estas ações tanto por parte dos cirurgiões-dentistas, quanto pelos médicos, apontam uma possível falha na formação clinica desses profissionais ${ }^{26}$. Com isso, fica claro a importância da participação dos graduandos e pós-graduandos da instituição de ensino superior no serviço de saúde, uma vez que os aproxima das gestantes, da realidade dos serviços de saúde, promovendo aprendizado para ambas as partes.

Neste estudo, algumas limitações podem ser destacadas, dentre elas, a composição da amostra, que foi constituída apenas por gestantes que concluíram o tratamento. Além disso, a dependência da resposta fiel das gestantes e o sentimento de gratidão $\log$ o após conclusão do tratamento podem ter interferido nas respostas. Por se tratar de uma instituição que oferece tratamento gratuito para as gestantes, grande parte apresenta baixas condições socioeconômicas, podendo acarretar em falta de senso crítico e um maior conformismo em relação aos serviços oferecidos ${ }^{17,29,30}$. Novas pesquisas com outras abordagens metodológicas podem ser realizadas.

\section{CONCLUSÃO}

O Programa de Atenção odontológica às Gestantes foi avaliado positivamente, a qualidade do serviço prestado pode ser considerada satisfatória em função da avaliação realizada. A maioria apresentou intenção de amamentar, comprovando que o programa contribui para a promoção da prática do aleitamento materno.

\section{REFERÊNCIAS}

1. Reis DM, Pitta DR, Ferreira HMB, Jesus MCP, Moraes MEL, Soares MG. Educação em saúde como estratégia de promoção de saúde bucal em gestantes. Cien Saude Coletiva. 2010; 15(1):269-76.

2. Pinheiro Filho JDS. Baixa Adesão e Dificuldade na Continuidade do Tratamento Odontológico na Gravidez, na Unidade Básica de Saúde Parque do Sol, em Campo Grande-MS. Monografia [Especialização em Atenção Básica em Saúde da Família]. Universidade Federal do Mato Grosso do Sul; 2011.

3. Bastiani C, Cota ALS, Provenzano MGA, Fracasso MDLC, Honório HM, Rios D. Conhecimento das gestantes sobre alterações bucais e tratamento odontológico durante a gravidez. Odontol Clín-Cient. 2010; 9(2):155-60.

4. Pesquisa Nacional de Saúde. Acesso e Utilização dos Serviços de Saúde, Acidentes e Violências. Instituto Brasileiro de Geografia e Estatística - IBGE, 2013.

5. Albuquerque OMRD, Abegg $\mathrm{C}$, Rodrigues CS. Percepção de gestantes do Programa de Saúde da Família em relação a barreiras no atendimento odontológico em Pernambuco, Brasil. Cad Saúde Pública. 2004; 20:(3)789-96.

6. Codato LAB, Nakama L, Melchior R. Percepções de gestantes sobre atenção odontológica durante a gravidez. Cien saude coletiva. 2008; 13(3):1075-80.

7. Santos Neto ET, Oliveira AE, Zandonade E, Leal MC. Acesso à assistência odontológica no acompanhamento pré-natal. Cien saude coletiva. 2012; 17(11):3057-68.

8. Codato LAB, Nakama L, Cordoni Jr L, Higasi MS. Atenção odontológica à gestante: papel dos profissionais de saúde. Cien saude coletiva. 2011; 16(4):2297-301.

9. Medeiros UV, Zevallos EFP, Rosiangela K. Promoção da saúde bucal da gestante: garantia de sucesso no futuro. Rev Cient do CRO-RJ. 2000; 2:47-57, 2000.

10. Lopes FF, Ribeiro TV, Fernandes DB, Calixto NRDV, Alves CMC, Pereira ALA et al. Oral health knowledge and practices among pregnant women using health services in São Luís, Maranhão, Brazil, 20072008. Epidemiol Serv Saúde. 2016; 25(4):819-26.

11. Hartz ZM, Pouvourville GD. Avaliação dos programas de saúde: a eficiência em questão. Cien saude coletiva. 1998; 3(1):68-82.

12. Brasil. DataSUS. Avaliação de Programas de SaúdeGoverno Federal do Brasil. PNASS - Programa Nacional 
de Avaliação de Serviços de Saúde.

13. Moimaz SAS, Rós DT, Bordin D, Rovida TAS, Garbin CAS. Satisfação e perfil de usuários do serviço odontológico no Sistema Único de Saúde. RFO - Passo Fundo. 2015; 20(3):334-39.

14. Brasil. Ministério da Saúde. Secretaria de Atenção à Saúde. Departamento de Ações Programáticas Estratégicas. Política nacional de atenção integral à saúde da mulher: plano de ação 2004-2007 / Ministério da Saúde, Secretaria de Atenção à Saúde, Departamento de Ações Programáticas Estratégicas - Brasília: Ministério da Saúde, 2004.

15. Diario da União. Resolução n 183, de 22 de setembro de 2017.Infraestrutura de Chaves Públicas Brasileira - ICPBrasil.

16. Ministério da Saúde. Secretaria de Atenção a Saúde. Departamento de Atenção Básica. Programa Nacional de Melhoria do Acesso e da Qualidade da Atenção Básica (PMAP) Manual Instrutivo - Anexo Ficha de Qualificação dos Indicadores.

17. Bordin D, Fadel CB, Moimaz SAS, Garbin CAS, Saliba NA. Comparative study of satisfaction of users and health professionals with the public dental service. Cien saude coletiva. 2017; 22(1):151-60.

18. Brasil. Ministerio da Saúde. Programa Nacional de Melhoria do Acesso e da Qualidade da Atenção Básica (PMAQ).

19. Kalil IR, Aguiar AC. Protagonista da amamentação ou instrumento da política de saúde infantil: a enunciação da mulher nos materiais oficiais de promoção e orientação ao aleitamento materno. Saúde Soc. 2016; 25(1):31-42.

20. Oliveira IM, Conde-Júnior AM, Cavalcante MMAS, Silva ABS, Rizzo MS, Leite C MC. Saberes Maternos Sobre a Relação da Amamentação Natural e Hábitos Bucais Deletérios. J Health Sci 2016; 18(2):75-9

21. Silva AE, Campos COM, Oliveira MCF, Ribeiro AQ, Cotta RMM, Araújo RMA. Changes in maternal conception about breastfeeding. Rev Bras Saúde Mater Infant. 2016; 16(4):399-406.

22. Moimaz SAS, Garbin CAS, Rocha NB, Santos SMG, Saliba NA. Resultados de dez anos do Programa de Atenção Odontológica à Gestante. Rev Ciênc Ext. 2011; 7(1):42-56.

23. Konishi F, Abreu e Lima F. Odontologia intra-uterina: a construção da saúde bucal antes do nascimento. Rev bras odontol. 2002; 59(5):294-5.

24. Moimaz SAS, Gomes AMP, Bordin D, Garbin CAS, Saliba NA. Extensão universitária como ferramenta geradora de ensino-aprendizagem e produtora de pesquisa. Rev Conex UEPG. 2015; 11(2):140-9.

25. Moimaz SAS, Saliba NA., Bino LDS, Rocha NB. A ótica do usuário na avaliação da qualidade do programa de atenção odontológica à gestante. Pesq Bras Odontoped Clin Integr. 2009; 9(2):147-53.

26. Trevisan CL, Pinto AAM. Fatores que interferem no acesso e na adesão das gestantes ao tratamento odontológico. Arch Health Invest. 2013; 2(2):29-35.

27. Brasil. Ministério da Saúde. Política Nacional de Humanização: a humanização como eixo norteador das práticas de atenção e gestão em todas as instâncias do SUS.Brasília, 2004.

28. Moimaz SAS, Rocha NB, Saliba O, Garbin CAS. O acesso de gestantes ao tratamento odontológico. Rev odontol univ cid Sao Paulo. 2007; 19(1):39-45.

29. Brandão ALRB, Giovanella L, Campos CEA. Avaliação da atenção básica pela perspectiva dos usuários: adaptação do instrumento EUROPEP para grandes centros urbanos brasileiros. Ciênc saude coletiva. 2013; 18(1):103-14.
30. Moimaz SAS, Burili MC, Bordin D, Garbin CAS, Saliba TA, Saliba NA. Satisfação dos usuários segundo variáveis de organização dos serviços públicos odontológicos. Arch Health Invest. 2017; 6(1):50-61.

\section{CONFLITO DE INTERESSES}

Os autores declaram não haver conflitos de interesse.

\section{AUTOR PARA CORRESPONDÊNCIA}

Suzely Adas Saliba Moimaz

sasaliba@foa.unesp.br 